\title{
Correspondence
}

\section{Complication of a non-invasive blood pressure cuff during prone positioning}

To the Editor:

Textbooks ascribe negligible risk to standard noninvasive blood pressure (NIBP) devices. ${ }^{1,2}$ We report a complication from an appropriately placed cuff. Consent for publication was obtained in conformance with institutional publication consent guidelines.

The cuff was placed on an athletic male and used during supine induction, then immediately inactivated after radial arterial catheter insertion and prone-positioning for a six-hour procedure. Postoperatively, marked erythema of the NIBP site and pronounced swelling of the proximal upper arm was observed. Painful, stocking-glove numbness in the dorsum of the hand and fingers presented. Orthopedic consultation confirmed significant edema of the upper extremity and compressive neurapraxia, which resolved spontaneously by the next day.

Standard NIBP placement occurred with the arm in full extension. After assuming the prone position, the arm was flexed at the elbow, increasing biceps muscle diameter. This is typically tolerated on a daily basis without adverse effects. With this athletic patient, however, critical constriction occurred beneath the non-cycled cuff with flexion, leading to the signs and symptoms described. While a rare complication, cuff placement cannot be assumed to be innocuous, particularly in limbs subsequently flexed, even with inactivated cuffs. Forearm cuff placement may be preferred in such instances, as diameter is not subjected to such significant changes with positioning. Occilometric measurements do not require placement on the upper arm, and smaller cuffs can be used with smaller diameter locations. We report for the first time a significant tourniquet effect from an appropriately applied NIBP cuff, occurring intraoperatively during prolonged non-utilization, and while in the prone position.

Paul M. Kempen MD PhD

Li Meng MD MPH

University of Pittsburgh, Presbyterian University

Hospital, Pittsburgh, USA

E-mail: kempenpm@anes.upmc.edu

Accepted for publication August 29, 2005.

\section{References}

1 Lake CL. Monitoring of arterial pressure. In: Lake C (Ed.).Clinical Monitoring for Anesthesia and Critical Care, $2^{\text {nd }}$ ed. Philadelphia, PA: WB Saunders; 1994: 101-18

2 Good ML, Gravenstien N. Anesthesia equipment. In: Gravenstein N, Kirby RR (Eds). Complications in Anesthesiology, $2^{\text {nd }}$ ed. Philadelphia, PA: LippincottRaven; 1996: 55-73.

\section{Cuffed versus uncuffed pediatric endotracheal tubes}

To the Editor:

We read with interest the editorial by Dr. Cox on cuffed vs uncuffed endotracheal tubes in children, ${ }^{1}$ and offer the following comments.

While there are advantages to using cuffed tubes for laparoscopic surgery, the small margin of safety associated with cuffed pediatric tube placement ${ }^{2}$ (a problem partially solved by the Microcuff $®$ Pediatric Tube $)^{3}$ is further reduced during laparoscopy, especially with the child in Trendelenberg position. The cephalad movement of the carina relative to the tube in those situations has led to endobronchial intubation in adults ${ }^{4}$ and children. For this reason, if a cuffed tube is used during laparoscopy, we believe that the "ideal" position of the cuff being distal to the cricoid cartilage $^{5}$ is unrealistic; instead, it should be passed just distal to the vocal cords. Conversely, when a child needs to be positioned prone, provision should be made for the tendency of the tube to move cephalad relative to the trachea. ${ }^{6}$

We agree with Dr. Cox that pulmonary compliance is an important consideration in the choice of tubes. Indeed, pediatric burn victims intubated with uncuffed tubes not uncommonly require tube change because of gas leak. ${ }^{7}$

For the many cases in which the choice of cuffed or uncuffed endotracheal tube makes little difference, and there is not a recent intubation record to act as a reference, it is more cost-effective to use a cuffed tube. The reason is that even though uncuffed tubes typically cost $10 \%$ less than cuffed tubes, $23 \%{ }^{8}$ of uncuffed tubes need to be changed because of poor fit ( $1 \%$ for cuffed tubes $\left.{ }^{8}\right)$. The difference is small, but the extra 
time incurred by tube change and the higher gas flow required when using uncuffed tubes ${ }^{8}$ also adds cost, not to mention the stress of tube change on the patient. Finally, there is always a higher theoretical risk of dispersion of infectious droplets when ventilating with an uncuffed tube a child with a highly communicable respiratory ailment. This was a consideration during the SARS outbreak in Hong Kong in 2003.

The availability of cuffed pediatric endotracheal tubes adds versatility to anesthesia practice. There is no doubt that cuffed pediatric tracheal tubes will continue to improve and their full potential will be realized. Meanwhile, we agree with Dr. Cox that the choice between cuffed and uncuffed pediatric endotracheal tubes should not be routine; that is just another reason why pediatric anesthesia can be so challenging and interesting.

Anthony M.-H. Ho MD FRCPC FCCP

Manoj K. Karmakar MD FRCA

The Chinese University of Hong Kong, Hong Kong, SAR

E-mail: hoamb@cuhk.edu.hk

Accepted for publication September 9, 2005.

\section{References}

$1 \operatorname{Cox} R G$. Should cuffed endotracheal tubes be used routinely in children? (Editorial). Can J Anesth 2005; 52: 669-74.

2 Ho AM, Aun CS, Karmakar MK. The margin of safety associated with the use of cuffed paediatric tracheal tubes. Anaesthesia 2002; 57: 173-5.

3 Weiss $M$, Balmer $C$, Dullenkopf $A$, et al. Intubation depth markings allow an improved positioning of endotracheal tubes in children. Can J Anesth 2005; 52: 721-6.

4 MacKenzie M, MacLeod K. Repeated inadvertent endobronchial intubation during laparoscopy. Br J Anaesth 2003; 91: 297-8.

5 James $I$. Cuffed tubes in children (Letter). Paediatr Anaesth 2001; 11: 259-63.

6 Marcano BV, Silver P, Sagy M. Cephalad movement of endotracheal tubes caused by prone positioning pediatric patients with acute respiratory distress syndrome. Pediatr Crit Care Med 2003; 4: 186-9.

7 Silver GM, Freiburg C, Halerz M, Tojong J, Supple K, Gamelli RL. A survey of airway and ventilator management strategies in North American pediatric burn units. J Burn Care Rehabil 2004; 25: 435-40.

8 Khine HH, Corddry DH, Kettrick RG, et al. Comparison of cuffed and uncuffed endotracheal tubes in young children during general anesthesia. Anesthesiology 1997; 86: 627-31.

\section{Proper preparation of the Trachlight ${ }^{T M}$ and endotracheal tube to facilitate intubation}

To the Editor:

While the Trachlight ${ }^{\mathrm{TM}}$ (Laerdal Medical Corp., Wappingers Falls, NY, USA) has been shown to be an effective and safe device for tracheal intubation, ${ }^{1}$ occasionally following the retraction of the internal stiff stylet, the tip of the endotracheal tube (ETT) seems to get "hung up" and cannot be readily advanced into the trachea. This is likely due to the fact that when an ETT is loaded along its natural curvature onto the Trachlight ${ }^{\mathrm{TM}}$, the tip of the ETT has a tendency to bend anteriorly upon retraction of the internal stiff stylet (Figure A). With this anterior bending, the tip of the ETT may be trapped or pushed against the anterior aspect of the cricoid ring or tracheal cartilaginous rings, making it difficult to further advance the ETT into the trachea. We would like to report two modified preparations of the Trachlight ${ }^{\mathrm{TM}}$ and ETT which may help to overcome this difficulty.

Firstly, immersing the ETT in warm saline solution prior to tracheal intubation will reduce its stiffness and the memory of its natural curvature, thus facilitating the advancement of the ETT into the trachea. Secondly, reverse loading of the ETT onto the Trachlight ${ }^{\mathrm{TM}}$ may minimize the tendency of the ETT tip to bend anteriorly while retracting the internal stiff stylet of the Trachlight ${ }^{\mathrm{TM}}$ (Figure B). With the reverse loading, the tip of the ETT is more likely to be directed down the trajectory of the trachea, making it easier to advance. The combination of softening and reverse loading of the ETT may potentially overcome the problems with "hang up" during intubation with the Trachlight ${ }^{\mathrm{TM}}$.

Over the last six months, we have used these modified preparations of the Trachlight ${ }^{\mathrm{TM}}$ and ETT for successful tracheal intubation in 160 patients. The "hang up" occurred in only two of the intubations (1.25\%). This contrasts with our previous experience (from the $\log$ book of one of the authors, ORH) in which a "hang up" during Trachlight ${ }^{\mathrm{TM}}$ intubation occurred in 12 of 82 patients (14.6\%). Thus, it appears that softening and reverse loading of the ETT can help to overcome the "hang up" while using the Trachlight ${ }^{\mathrm{TM}}$.

It should be emphasized that this concept of reverse loading of the ETT is not a novel one. Similar reverse loading has previously been described with the use of a regular ETT for intubation through the Fastrach (intubating) laryngeal mask airway. Joo et al. found that blind tracheal intubation through the Fastrach can 